Review Article

\title{
Gray Matter Atrophy within the Default Mode Network of Fibromyalgia: A Meta-Analysis of Voxel-Based Morphometry Studies
}

\author{
Chemin Lin, ${ }^{1,2}$ Shwu-Hua Lee, ${ }^{2,3}$ and Hsu-Huei Weng ${ }^{4,5,6,7,8}$ \\ ${ }^{1}$ Department of Psychiatry, Keelung Chang Gung Memorial Hospital, Keelung, Taiwan \\ ${ }^{2}$ College of Medicine, Chang Gung University, Taoyuan, Taiwan \\ ${ }^{3}$ Department of Psychiatry, Linkou Chang Gung Memorial Hospital, Taoyuan, Taiwan \\ ${ }^{4}$ Department of Radiology, Chiayi Chang Gung Memorial Hospital, Chiayi, Taiwan \\ ${ }^{5}$ Department of Diagnostic Radiology, Chiayi Chang Gung Memorial Hospital, \\ Chang Gung University College of Medicine, Chiayi, Taiwan \\ ${ }^{6}$ Department of Respiratory Care, Chang Gung University of Science and Technology, Chiayi, Taiwan \\ ${ }^{7}$ Department of Psychology, National Chung Cheng University, Chiayi, Taiwan \\ ${ }^{8}$ Department of Imaging Physics, Division of Diagnostic Imaging, University of Texas, \\ MD Anderson Cancer Center, Houston, TX, USA
}

Correspondence should be addressed to Shwu-Hua Lee; shl@adm.cgmh.org.tw and Hsu-Huei Weng; hweng@post.harvard.edu

Received 16 September 2016; Accepted 6 December 2016

Academic Editor: Sadiq Umar

Copyright ( 2016 Chemin Lin et al. This is an open access article distributed under the Creative Commons Attribution License, which permits unrestricted use, distribution, and reproduction in any medium, provided the original work is properly cited.

Over the years, studies have demonstrated morphological changes in the brain of fibromyalgia (FMS). We aimed to conduct a coordinate-based meta-analytic research through systemic review on voxel-based morphometry (VBM) imaging results to identify consistent gray matter (GM) difference between FMS patients and healthy subjects. We performed a comprehensive literature search in PubMed (January 2000-December 2015) and included six VBM publication on FMS. Stereotactic data were extracted from 180 patients of FMS and 123 healthy controls. By means of activation likelihood estimation (ALE) technique, regional GM reduction in left medial prefrontal cortex and right dorsal posterior cingulate cortex was identified. Both regions are within the default mode network. In conclusion, the gray matter deficit is related to the both affective and nonaffective components of pain processing. This result also provided the neuroanatomical correlates for emotional and cognitive symptoms in FMS.

\section{Introduction}

Fibromyalgia (FM) is characterized by chronic widespread pain with accompanying symptoms, such as fatigue, morning stiffness, insomnia, cognitive dullness, depression, and anxiety $[1,2]$. Not only does FM impair life quality [3], but it also increases disability and absence from work [4]. With the evolving diagnostic criteria of FM [5], the prevalence rate is estimated about $2 \sim 4 \%$ in the general population with female predominance $[6,7]$. Despite genetic, environmental, and biochemical factors proposed as the underlying pathophysiology $[8,9]$, the exact mechanism pertaining to FM is still under debate. Early research on peripheral neural or muscular damage in patients of FM failed to detect consistent evidence [10]. Therefore, central sensitization has been proposed to explain the features of FM, including allodynia (increased sensitivity to stimulus that does not normally provoke pain) and hyperalgesia (heightened response to painful stimulus) $[11,12]$.

Over the past two decade, central sensitization theory can be further exemplified in the studies using neuroimaging techniques. Single-photon-emission-computed tomography (SPECT) imaging has demonstrated regional cerebral blood flow $(\mathrm{rCBF})$ decrease in thalamus and pontine region in 
patients of FM $[13,14]$. The ensuing PET study has not only shown rCBF decrease in several brain regions, supporting the dysfunctional cognitive processing of pain, but also higher retrosplenial rCBF, suggesting secondary hyperalgesia [15]. Since the inception of functional magnetic resonance imaging ( $\mathrm{fMRI}$ ), it became easier to infer neural activity by proxy of blood-oxygen-level-dependent (BOLD) contrast without the need of radioactive tracer or contrast agent. Multiple paradigms were applied to study the differential activation between FM patients and normal controls. With the stimuli of pressure [16-18], repetitive heat pulses [19], nociceptive injection [20], or even incision of forearm [21], compared to normal, FM patients showed increased activation in assorted pain related brain areas, such as thalamus, primary (S1) as well as secondary (S2) somatosensory cortices, insula, and cingulate cortex [16-21]. On the other hand, impaired descending inhibitory pain system was evinced from the results of decreased activation in rACC (rostral anterior cingulate cortex) and thalamus [18] and reduced resting-state functional connectivity between ACC with PAG (periaqueductal gray) [22] in FM patients.

Although tasked-related fMRI helps us extract specific cognitive function in FM patients, the variety of different paradigms impede us from compiling or comparing all these studies directly. Besides functional changes, research on structural changes in chronic pain patients has concurrently been in the limelight. Voxel-based morphometry (VBM) [23] is the most widely employed technique and has been implicated in several groups, including chronic low back pain [24, 25], tension headache [26], irritable bowel syndrome [27], and chronic pelvic pain [28]. In fibromyalgia, several articles using region-of-interest (ROI) method focusing on "pain matrix" in brain have discovered gray matter decrease in anterior [29-31] and midcingulate [29] cortices, prefrontal cortex [30], midinsula [29], amygdala [30, 31], right and left lateral orbitofrontal cortex [31], and hippocampi [32].

These studies reiterated the theoretical importance of the key brain regions. However, unbiased whole-brain studies could also be conducted by VBM without need of selected ROIs. Furthermore, by using meta-analytic approach of activation likelihood estimate (ALE) method [33, 34], we are able to pinpoint consistent structural brain changes in several illness, such as schizophrenia [35], bipolar disorder [36], posttraumatic stress disorder [37], Alzheimer's disease [38], narcolepsy [39], chronic pain [40], and headache [41]. Therefore, to provide greater insight into the structure changes of FM, current study aims to review the findings from all the whole-brain VBM research published to date and to conduct an ALE-based meta-analysis. We also hypothesize that we could observe convergent brain areas associated with the features FM encompasses.

\section{Methods and Materials}

2.1. Search Strategies and Selection Criteria. We conducted a comprehensive literature search in PubMed database (January 2000-December 2015) by using the key works "fibromyalgia," "voxel," "morphometry," "voxel-based morphometry," and "VBM." We did not set specific restrictions on language of the literature. Cited articles in systemic reviews related to brain structure changes in fibromyalgia were also examined to increase any possibility of unincluded papers in previous literature search. Two independent investigators ( $\mathrm{LC}$ and $\mathrm{WHH}$ ) evaluated the methodology and the quality of the selected studies, where demographics, name of the first author, publication year, title of the journal, age of the participants, total number of participants, sex ratio, matching method, and the methods and thresholds in SPM were also examined.

\subsection{Study Selection}

2.2.1. Inclusion Criteria. The inclusion criteria for the studies in this meta-analysis are (1) peer-reviewed articles with retrievable full-text, (2) patients were diagnosed with fibromyalgia with healthy controls, (3) VBM procedure in MR anatomical analysis done in whole-brain structure by either GMV or GMC; we excluded region-of-interest (ROI) or volume-of-interest (VOI) method, and (4) peak coordinates available in Montreal Neurologic Institute (MNI) or Talairach and Tournoux stereotactic space.

2.2.2. Data Extraction. We inputted the $x, y$, and $z$ peak activation coordinates from the eligible contrasts into the meta-analysis. Coordinates reported in MNI space were converted to Talairach coordinates [42, 43]. We texted and employed the MNI coordinates from the data to GingerALE 2.1.1 (http://brainmap.org/ale/, Research Imaging Institute of the University of Texas Health Science Center, San Antonio, TX).

2.2.3. ALE Meta-Analysis. The version of current ALE approach $[33,44,45]$ is the one implemented from the CBMA of neuroimaging results $[34,45]$. In short, each stereotactic coordinates were represented as the peak center of the three-dimensional (3D) Gaussian probability distribution. A computed modeled activation (MA) map was derived to encapsulate the localization probabilities. The sample size of each study was used to estimate the spatial uncertainty related to the activation [46]. The overlapping of the distributions across different studies was calculated. We calculated the ALE values voxel-by-voxel by summing the MA maps derived from above. In doing so, the current analysis would not deal with foci (fixed effects), but with the concurrence from the studies (random effects). We set a 0.05 false discovery rate (FDR; q) for multiple comparisons correction $[34,47]$ with a minimum cluster size $200 \mathrm{~mm}^{3}$. We created NIfTI (.nii) format in our output image and displayed result with Mango (Multi-Image Analysis Graphical User Interface [GUI]), along with MNI space Colin brain template [48]. We labeled the clusters by using SPM Anatomy Toolboxvl.8 (http://www.fz-juelich.de/inm/inm-1/DE/Forschung/_docs/SPMAnatomyToolbox/SPMAnatomyToolbox node.html) $[46,49,50]$.

\section{Results}

Based on the inclusion criteria, 7 VBM studies [51-57], in which 8 groups of comparison between FMS patients and 

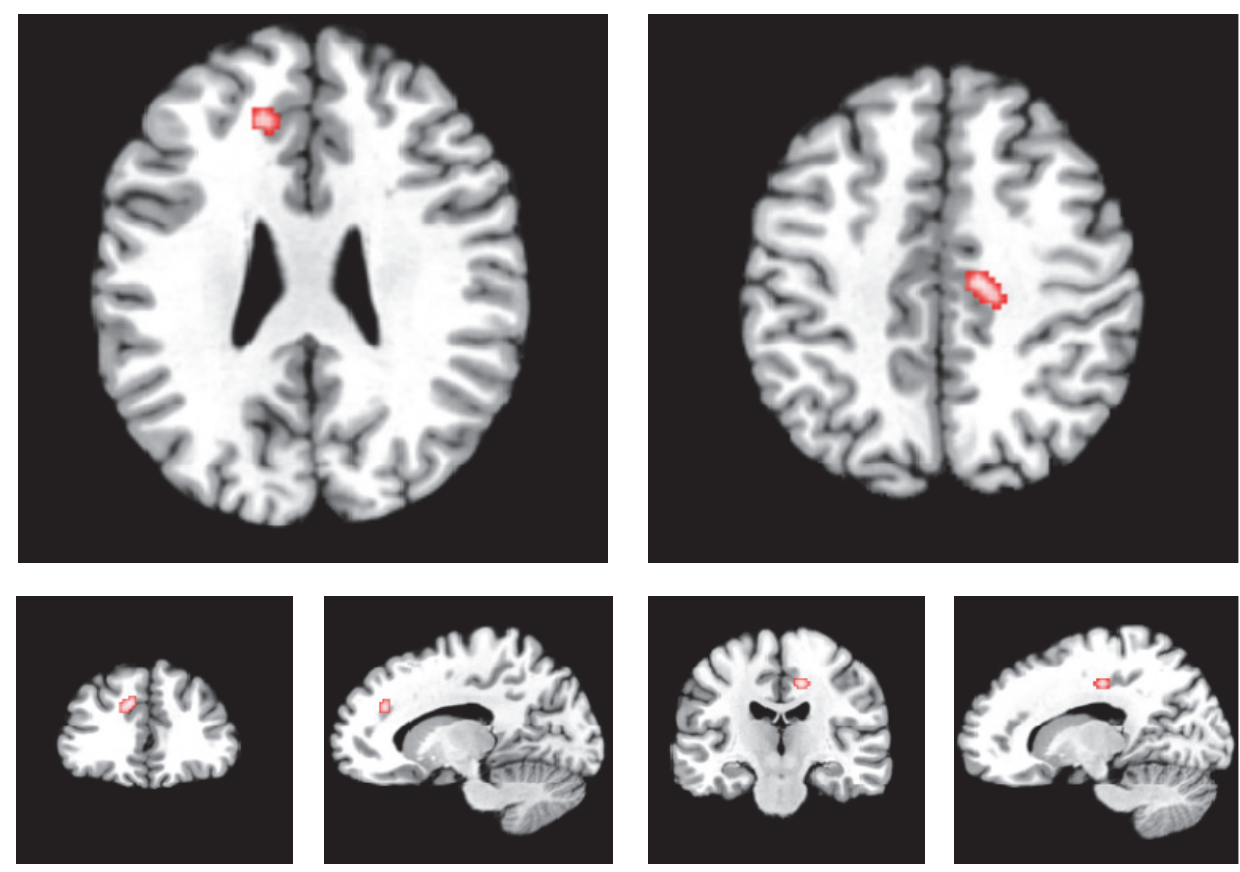

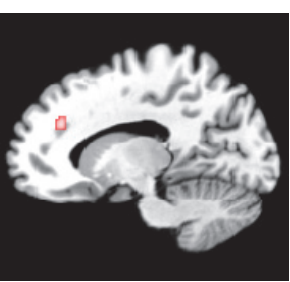

(a)

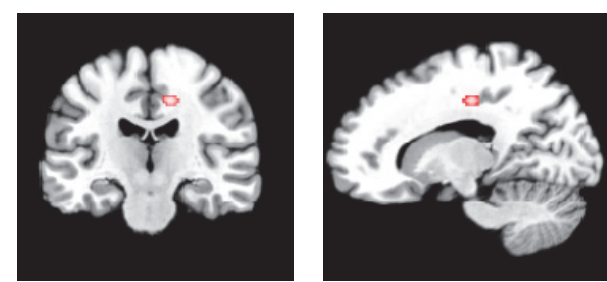

(b)

FIGURE 1: Sagittal, coronal, and axial sections of regions of gray matter reduction in fibromyalgia compared with normal controls ((a) left medial prefrontal cortex and (b) right dorsal posterior cingulate cortex). Results are from the activation likelihood estimation. Activation is significant at $p<0.05$ corrected for multiple comparisons using the false discovery rate.

controls, were eligible in the final analysis (Table 1). In sum, 180 fibromyalgia patients and 123 controls were included. A total of 47 peak coordinates were reported for brain structural changes, of which 31 were included for decreased gray matter and 16 were included for increased gray matter, in patients of FMS compared with NC. The ALE metaanalysis revealed gray matter decreased in patients of FMS in 2 clusters (illustrated in Figure 1 and Table 2), including left medial prefrontal cortex, extending partly to anterior cingulate area (mPFC/ACC, BA 9/32, cluster size: $432 \mathrm{~mm}^{3}$, and coordinates of cluster maxima: $x:-10, y: 34, z: 30$ ) and right dorsal posterior cingulate cortex, extending to posterior-midcingulate cortex (dPCC/pMCC, BA 31, cluster size: $368 \mathrm{~mm}^{3}$, and coordinates of cluster maxima: $x: 12, y$ : $-18, z: 42)$. We adopted the classification proposed by Vogt and Destrieux $[58,59]$ in the subdivisions of the cingulate cortex. No foci survived in the analysis showing gray matter increases in the reverse contrast.

\section{Discussion}

We employed meta-analytic analysis with ALE to pool VBM studies to assess the structural brain changes between FM patients and healthy subjects. We identified the patients of FM to have GM reduction in left $\mathrm{mPFC}$ and right dPCC.

$\mathrm{mPFC}$ has been denoted as part of the medial pain system $[60,61]$. Stimulation of mPFC with transcranial magnetic stimulation (TMS) pulse may increase sensitivity [62] and decrease threshold of pain perception [63]. In another singlephoton emission computed tomography (SPECT) study, the deactivation of mPFC after repetitive TMS over the primary motor cortex was correlated with pain reduction [64]. Thus, $\mathrm{mPFC}$ has been postulated to be involved in the descending pain modulation system $[65,66]$, whether inhibitory or facilitatory. Therefore, our result may be supportive to the proposed mechanism of the dysfunctional descending analgesia in FMS, rendering the chronic widespread pain $[67,68]$.

Besides pain, neuropsychological symptoms, including depression, anxiety [69], and cognitive complaints [70], are frequent in FMS. As catastrophizing may amplify pain processing [71], it is found that brain activity in mPFC is correlated with catastrophizing [72]. A plausible explanation is that, along with ACC, $\mathrm{mPFC}$ is the brain region activated by the anticipation of pain [73] and serves as motivational aspect of pain [74]. Moreover, the morphology in $\mathrm{mPFC}$ is negatively correlated with performance in working memory and positively correlated with pain perception [75], a finding that signifies the pain-cognition interaction. In sum, MPFC is the brain region known to modulate cognitive and emotional processing. Along the caudal-rostral axis of mPFC, objective, subject, and even meta-cognitive aspects of pain are represented [76]. It may be these varied functions of mPFC to account for the diverse symptoms in FMS.

Another area that exhibited decreased GM volume in FMS was right dorsal PCC. In the more specific breakdown of this cluster in our study, it actually comprises of dPCC and pMCC. Dorsal (rostral) PCC is associated with pain in metaanalysis [77], and pMCC is the region linked with several chronic somatic pain disorders [78]. Unlike the involvement 


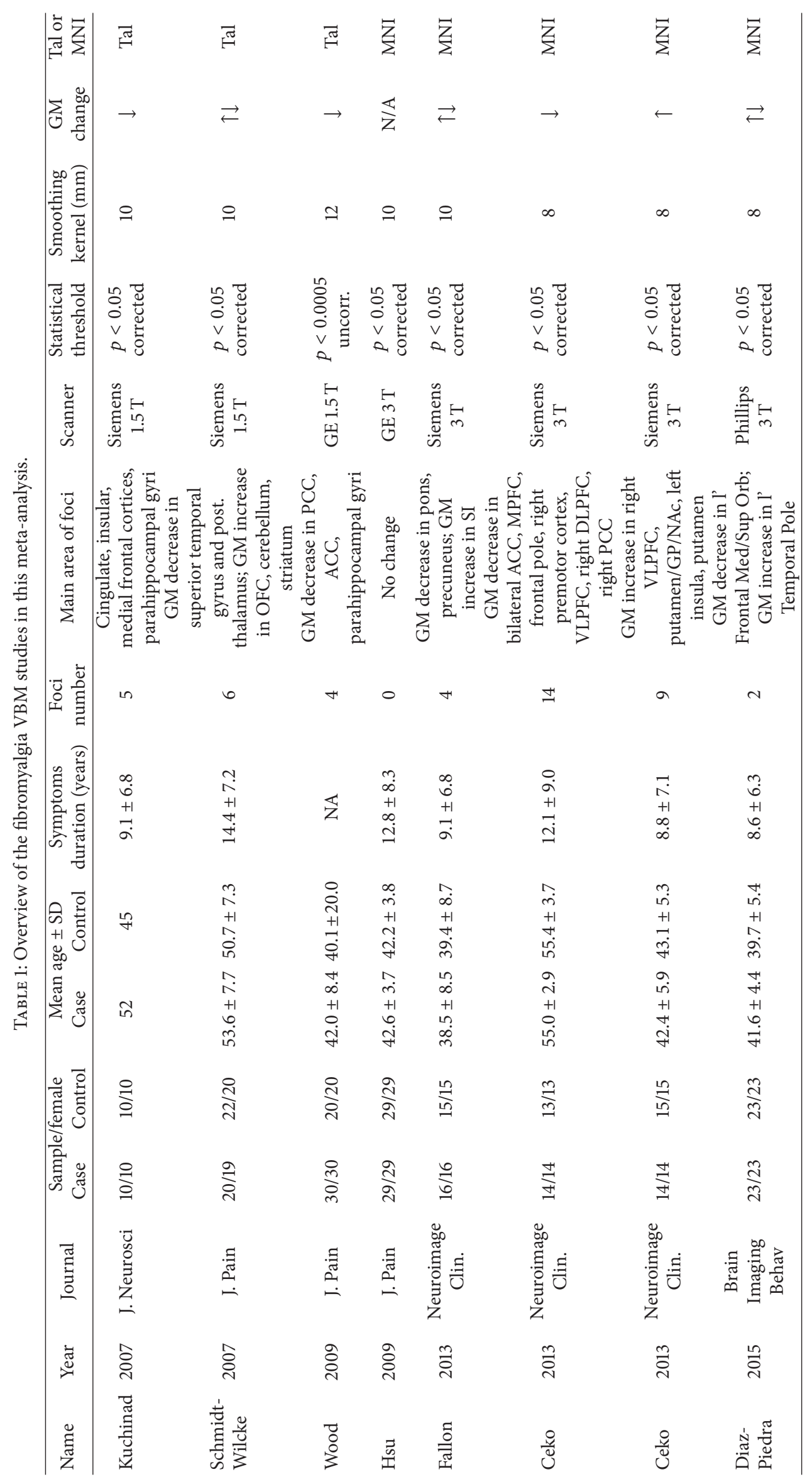


TABLE 2: Regions of gray matter reduction in fibromyalgia relative to healthy controls.

\begin{tabular}{lcccccc}
\hline \multirow{2}{*}{ Region } & \multirow{2}{*}{ BA } & \multicolumn{3}{c}{ MNI coordinates } & ALE extrema value & Cluster size $\left(\mathrm{mm}^{3}\right)$ \\
\hline Left medial prefrontal cortex & $9 / 32$ & -10 & 34 & 30 & 0.0103 & 432 \\
Right dorsal posterior cingulate cortex & 31 & 12 & -18 & 42 & 0.0101 & 368 \\
\hline
\end{tabular}

BA: Brodmann area; MNI: Montreal Neurological Institute; ALE: activation likelihood estimation.

of $\mathrm{mPFC}$ and ACC in the emotional component of pain processing [79], dPCC/pMCC is related to orienting the body toward nocuous stimuli, the nonaffective component of pain processing [59], and contributes to the prepotent withdrawal reaction from pain [80]. Damage of dPCC/pMCC could lead to visuospatial damage [81].

While clock drawing test has been employed as a screening tool for cognitive decline in FMS [82], visuospatial memory impairment has been denoted as the early signal of cognitive decline in FMS, even in ahead of verbal memory decline $[83,84]$. Our result may provide the neural underpinning for this domain of cognitive deficit in FMS. Besides its role in orienting motor response, $\mathrm{dPCC} / \mathrm{pMCC}$ is also the initial region demonstrating damage in mild cognitive impairment [81]. Along with MPFC, PCC is involved in self-referential mental activity [85] and plays a pivotal part in default mode network (DMN) [86]. Convergent evidences have reported reduced metabolic activities in PCC in early Alzheimer's disease [87], cognitive decline [88], and ageing [89]. Hence, GM decrease in PCC is attributable to the cognitive dysfunction frequently found in FMS [54] and is also supportive to the view that FMS accelerates premature ageing of the brain [51].

The finding of concurrent GM decrease in $\mathrm{mPFC}$ and dPCC in our result does not come in coincidence as they are among the key brain regions constituting DMN. The implication of this result could be inferred from previous restingstate functional connectivity brain imaging studies.

dPCC has been demonstrated with greater connectivity at rest with mPFC [90, 91]. Nevertheless, this connectivity is diminished in FMS [22]. Besides, DMN abnormality is associated with FMS [92] and chronic back pain [93]. At the same time, it has been suggested that greater activation of DMN helps people mind-wander away from pain [94]. Thus, we speculate that integrity of the DMN is necessary for ongoing cognitive modulation on pain processing and that its dysfunction may jeopardize nociception.

\section{Limitations}

Our study is based on meta-analytic approach with several inherent limitations. First, despite rigorous literature search, we included only 7 studies in the final analysis due to exclusion of those studies using ROI method and those without providing coordinates. In addition, our results may be subject to publication bias. Those with negative finding are less often published. Second, these included VBM studies are varied in their methods, such as difference in preprocessing steps (traditional or optimized), smoothing kernels, and statistical threshold. Besides, inclusion and exclusion criteria differ across the studies. This may also influence the individual result. Third, FMS is a complex disease itself with multiple comorbidities. A recent study found that each symptom dimension in FMS was related to a specific brain morphological change [57]. Another recent database research demonstrated a bidirectional association between FMS and depression [95]. We did not exclude those comorbid with depression specifically. Indeed, our result revealed GM decreases in mPFC, a common region reported both in depression $[96,97]$ and FMS $[54,56]$ research. As most pain related brain imaging studies, we cannot ascertain this structural change related to depression or pain itself [98]. In the same vein, to answer the question whether the GM decreases is a consequence of illness or the cause of the illness is out of the scope by current study design. Chronic nociceptive stimulus is thought as a cause for GM decreases [99] as some evidences have displayed correlation between pain duration [25]. Still, more longitudinal cohort studies are needed to disentangle this problem.

\section{Conclusions}

In conclusion, current meta-analytic results indicate the decrease gray matter volume in left $\mathrm{MPFC}$ and right dPCC in patients of FMS. These two areas are related to cognitive, affective, and nonaffective components of pain processing, supporting the notion of central sensitization theory of FMS. In addition, $\mathrm{mPFC}$ and right $\mathrm{dPCC}$ are also deeply linked with the default mode network. Thus, beside the areas of the typical "pain matrix," such as insula or anterior cingulate gyrus, our results may shed light on other area of the same importance and provide insight over future research on the mechanism of fibromyalgia.

\section{Disclosure}

The funders did not play any role in the study design, data collection and analysis, decision to publish, or preparation of the manuscript.

\section{Competing Interests}

The authors declare no conflict of interests.

\section{Authors' Contributions}

Chemin Lin, Shwu-Hua Lee, and Hsu-Huei Weng conceived the study design, Chemin Lin and Hsu-Huei Weng conducted the experiments, Chemin Lin wrote the paper, and HsuHuei Weng reviewed the analysis. All authors reviewed the manuscript. 


\section{Acknowledgments}

This work was supported by the Medical Research Grants from Chang Gung Memorial Hospital (CMRPG3C0043) to Chemin Lin.

\section{References}

[1] F. Wolfe, H. A. Smythe, M. B. Yunus et al., "The American College of Rheumatology 1990 criteria for the classification of fibromyalgia," Arthritis \& Rheumatism, vol. 33, no. 2, pp. 160$172,1990$.

[2] F. Wolfe, D. J. Clauw, M.-A. Fitzcharles et al., "The American College of Rheumatology preliminary diagnostic criteria for fibromyalgia and measurement of symptom severity," Arthritis Care \& Research, vol. 62, no. 5, pp. 600-610, 2010.

[3] J. A. Verbunt, D. H. F. M. Pernot, and R. J. E. M. Smeets, "Disability and quality of life in patients with fibromyalgia," Health and Quality of Life Outcomes, vol. 6, no. 1, article 8, 2008.

[4] M. Kivimäki, P. Leino-Arjas, L. Kaila-Kangas et al., "Increased absence due to sickness among employees with fibromyalgia," Annals of the Rheumatic Diseases, vol. 66, no. 1, pp. 65-69, 2007.

[5] M.-A. Fitzcharles, P. A. Ste-Marie, and J. X. Pereira, "Fibromyalgia: evolving concepts over the past 2 decades," Canadian Medical Association Journal, vol. 185, no. 13, pp. E645-E651, 2013.

[6] F. Wolfe, K. Ross, J. Anderson, I. J. Russell, and L. Hebert, “The prevalence and characteristics of fibromyalgia in the general population," Arthritis and Rheumatism, vol. 38, no. 1, pp. 19-28, 1995.

[7] F. Wolfe, E. Brähler, A. Hinz, and W. Häuser, "Fibromyalgia prevalence, somatic symptom reporting, and the dimensionality of polysymptomatic distress: results from a survey of the general population," Arthritis Care \& Research, vol. 65, no. 5, pp. 777-785, 2013.

[8] P. Mease, "Fibromyalgia syndrome: review of clinical presentation, pathogenesis, outcome measures, and treatment," The Journal of Rheumatology, vol. 75, pp. 6-21, 2005.

[9] D. J. Clauw, "Fibromyalgia: an overview," American Journal of Medicine, vol. 122, no. 12, pp. S3-S13, 2009.

[10] R. W. Simms, S. H. Roy, M. Hrovat et al., "Lack of association between fibromyalgia syndrome and abnormalities in muscle energy metabolism," Arthritis \& Rheumatism, vol. 37, no. 6, pp. 794-800, 1994.

[11] J. A. Desmeules, C. Cedraschi, E. Rapiti et al., "Neurophysiologic evidence for a central sensitization in patients with fibromyalgia," Arthritis \& Rheumatism, vol. 48, no. 5, pp. 14201429, 2003.

[12] R. Staud, "Evidence of involvement of central neural mechanisms in generating fibromyalgia pain," Current rheumatology reports, vol. 4, no. 4, pp. 299-305, 2002.

[13] J. M. Mountz, L. A. Bradley, J. G. Modell et al., "Fibromyalgia in women. Abnormalities of regional cerebral blood flow in the thalamus and the caudate nucleus are associated with low pain threshold levels," Arthritis and Rheumatism, vol. 38, no. 7, pp. 926-938, 1995.

[14] R. Kwiatek, L. Barnden, R. Tedman et al., "Regional cerebral blood flow in fibromyalgia: single-photon-emission computed tomography evidence of reduction in the pontine tegmentum and thalami," Arthritis and Rheumatism, vol. 43, no. 12, pp. 2823-2833, 2000.
[15] G. Wik, H. Fischer, B. Bragée, M. Kristianson, and M. Fredrikson, "Retrosplenial cortical activation in the fibromyalgia syndrome," NeuroReport, vol. 14, no. 4, pp. 619-621, 2003.

[16] T. Giesecke, R. H. Gracely, M. A. B. Grant et al., "Evidence of augmented central pain processing in idiopathic chronic low back pain," Arthritis \& Rheumatism, vol. 50, no. 2, pp. 613-623, 2004.

[17] R. H. Gracely, F. Petzke, J. M. Wolf, and D. J. Clauw, "Functional magnetic resonance imaging evidence of augmented pain processing in fibromyalgia," Arthritis \& Rheumatism, vol. 46, no. 5, pp. 1333-1343, 2002.

[18] K. B. Jensen, E. Kosek, F. Petzke et al., "Evidence of dysfunctional pain inhibition in Fibromyalgia reflected in rACC during provoked pain," Pain, vol. 144, no. 1-2, pp. 95-100, 2009.

[19] R. Staud, J. G. Craggs, W. M. Perlstein, M. E. Robinson, and D. D. Price, "Brain activity associated with slow temporal summation of C-fiber evoked pain in fibromyalgia patients and healthy controls," European Journal of Pain, vol. 12, no. 8, pp. 1078-1089, 2008.

[20] M. Diersl, M. T. Schleyl, M. Rancel et al., "Differential central pain processing following repetitive intramuscular proton/prostaglandin E2 injections in female fibromyalgia patients and healthy controls," European Journal of Pain, vol. 15, no. 7, pp. 716-723, 2011.

[21] M. Burgmer, E. Pogatzki-Zahn, M. Gaubitz, E. Wessoleck, G. Heuft, and B. Pfleiderer, "Altered brain activity during pain processing in fibromyalgia," Neurolmage, vol. 44, no. 2, pp. 502508, 2009.

[22] I. Cifre, C. Sitges, D. Fraiman et al., "Disrupted functional connectivity of the pain network in fibromyalgia," Psychosomatic Medicine, vol. 74, no. 1, pp. 55-62, 2012.

[23] J. Ashburner and K. J. Friston, "Unified segmentation," NeuroImage, vol. 26, no. 3, pp. 839-851, 2005.

[24] T. Schmidt-Wilcke, E. Leinisch, S. Gänbauer et al., "Affective components and intensity of pain correlate with structural differences in gray matter in chronic back pain patients," Pain, vol. 125, no. 1-2, pp. 89-97, 2006.

[25] A. V. Apkarian, Y. Sosa, S. Sonty et al., "Chronic back pain is associated with decreased prefrontal and thalamic gray matter density," Journal of Neuroscience, vol. 24, no. 46, pp. 10410-10415, 2004.

[26] T. Schmidt-Wilcke, E. Leinisch, A. Straube et al., "Gray matter decrease in patients with chronic tension type headache," Neurology, vol. 65, no. 9, pp. 1483-1486, 2005.

[27] D. A. Seminowicz, J. S. Labus, J. A. Bueller et al., "Regional gray matter density changes in brains of patients with irritable bowel syndrome," Gastroenterology, vol. 139, no. 1, pp. 48.e2-57.e2, 2010.

[28] S. As-Sanie, R. E. Harris, V. Napadow et al., "Changes in regional gray matter volume in women with chronic pelvic pain: a voxelbased morphometry study," Pain, vol. 153, no. 5, pp. 1006-1014, 2012.

[29] M. E. Robinson, J. G. Craggs, D. D. Price, W. M. Perlstein, and R. Staud, "Gray matter volumes of pain-related brain areas are decreased in fibromyalgia syndrome," Journal of Pain, vol. 12, no. 4, pp. 436-443, 2011.

[30] M. Burgmer, M. Gaubitz, C. Konrad et al., "Decreased gray matter volumes in the cingulo-frontal cortex and the amygdala in patients with fibromyalgia," Psychosomatic Medicine, vol. 71, no. 5, pp. 566-573, 2009. 
[31] K. B. Jensen, P. Srinivasan, R. Spaeth et al., "Overlapping structural and functional brain changes in patients with long-term exposure to fibromyalgia pain," Arthritis and Rheumatism, vol. 65, no. 12, pp. 3293-3303, 2013.

[32] J. Lutz, L. Jäger, D. de Quervain et al., "White and gray matter abnormalities in the brain of patients with fibromyalgia: a diffusion-tensor and volumetric imaging study," Arthritis \& Rheumatism, vol. 58, no. 12, pp. 3960-3969, 2008.

[33] S. B. Eickhoff, A. R. Laird, C. Grefkes, L. E. Wang, K. Zilles, and P. T. Fox, "Coordinate-based activation likelihood estimation meta-analysis of neuroimaging data: a random-effects approach based on empirical estimates of spatial uncertainty," Human Brain Mapping, vol. 30, no. 9, pp. 2907-2926, 2009.

[34] A. R. Laird, P. M. Fox, C. J. Price et al., "ALE meta-analysis: controlling the false discovery rate and performing statistical contrasts," Human Brain Mapping, vol. 25, no. 1, pp. 155-164, 2005.

[35] R. C. K. Chan, X. Di, G. M. McAlonan, and Q.-Y. Gong, "Brain anatomical abnormalities in high-risk individuals, first-episode, and chronic schizophrenia: an activation likelihood estimation meta-analysis of illness progression," Schizophrenia Bulletin, vol. 37, no. 1, pp. 177-188, 2011.

[36] E. Bora, A. Fornito, M. Yücel, and C. Pantelis, "Voxelwise meta-analysis of gray matter abnormalities in bipolar disorder," Biological Psychiatry, vol. 67, no. 11, pp. 1097-1105, 2010.

[37] S. Kühn and J. Gallinat, "Gray matter correlates of posttraumatic stress disorder: a quantitative meta-analysis," Biological Psychiatry, vol. 73, no. 1, pp. 70-74, 2013.

[38] L. K. Ferreira, B. S. Diniz, O. V. Forlenza, G. F. Busatto, and M. V. Zanetti, "Neurostructural predictors of Alzheimer's disease: a meta-analysis of VBM studies," Neurobiology of Aging, vol. 32, no. 10, pp. 1733-1741, 2011.

[39] H.-H. Weng, C.-F. Chen, Y.-H. Tsai et al., "Gray matter atrophy in narcolepsy: an activation likelihood estimation metaanalysis," Neuroscience \& Biobehavioral Reviews, vol. 59, pp. 5363, 2015.

[40] R. F. Smallwood, A. R. Laird, A. E. Ramage et al., "Structural brain anomalies and chronic pain: a quantitative meta-analysis of gray matter volume," Journal of Pain, vol. 14, no. 7, pp. 663675, 2013.

[41] A. May, "Morphing voxels: the hype around structural imaging of headache patients," Brain, vol. 132, no. 6, pp. 1419-1425, 2009.

[42] J. L. Lancaster, D. Tordesillas-Gutiérrez, M. Martinez et al., "Bias between MNI and talairach coordinates analyzed using the ICBM-152 brain template," Human Brain Mapping, vol. 28, no. 11, pp. 1194-1205, 2007.

[43] A. R. Laird, J. L. Robinson, K. M. McMillan et al., "Comparison of the disparity between Talairach and MNI coordinates in functional neuroimaging data: validation of the Lancaster transform," NeuroImage, vol. 51, no. 2, pp. 677-683, 2010.

[44] S. B. Eickhoff, D. Bzdok, A. R. Laird, F. Kurth, and P. T. Fox, "Activation likelihood estimation meta-analysis revisited," NeuroImage, vol. 59, no. 3, pp. 2349-2361, 2012.

[45] P. E. Turkeltaub, G. F. Eden, K. M. Jones, and T. A. Zeffiro, "Meta-analysis of the functional neuroanatomy of single-word reading: method and validation," NeuroImage, vol. 16, no. 3, part 1, pp. 765-780, 2002.

[46] S. B. Eickhoff, K. E. Stephan, H. Mohlberg et al., "A new SPM toolbox for combining probabilistic cytoarchitectonic maps and functional imaging data," NeuroImage, vol. 25, no. 4, pp. 13251335, 2005.
[47] C. R. Genovese, N. A. Lazar, and T. Nichols, "Thresholding of statistical maps in functional neuroimaging using the false discovery rate," NeuroImage, vol. 15, no. 4, pp. 870-878, 2002.

[48] C. J. Holmes, R. Hoge, L. Collins, R. Woods, A. W. Toga, and A. C. Evans, "Enhancement of MR images using registration for signal averaging," Journal of Computer Assisted Tomography, vol. 22, no. 2, pp. 324-333, 1998.

[49] S. B. Eickhoff, S. Heim, K. Zilles, and K. Amunts, "Testing anatomically specified hypotheses in functional imaging using cytoarchitectonic maps," NeuroImage, vol. 32, no. 2, pp. 570$582,2006$.

[50] S. B. Eickhoff, T. Paus, S. Caspers et al., "Assignment of functional activations to probabilistic cytoarchitectonic areas revisited," NeuroImage, vol. 36, no. 3, pp. 511-521, 2007.

[51] A. Kuchinad, P. Schweinhardt, D. A. Seminowicz, P. B. Wood, B. A. Chizh, and M. C. Bushnell, "Accelerated brain gray matter loss in fibromyalgia patients: premature aging of the brain?" The Journal of Neuroscience, vol. 27, no. 15, pp. 4004-4007, 2007.

[52] T. Schmidt-Wilcke, R. Luerding, T. Weigand et al., "Striatal grey matter increase in patients suffering from fibromyalgia-a voxel-based morphometry study," Pain, vol. 132, no. 1, pp. S109S116, 2007.

[53] M. C. Hsu, R. E. Harris, P. C. Sundgren et al., "No consistent difference in gray matter volume between individuals with fibromyalgia and age-matched healthy subjects when controlling for affective disorder," Pain, vol. 143, no. 3, pp. 262-267, 2009.

[54] P. B. Wood, M. F. Glabus, R. Simpson, and J. C. Patterson II, "Changes in gray matter density in fibromyalgia: correlation with dopamine metabolism," The Journal of Pain, vol. 10, no. 6, pp. 609-618, 2009.

[55] N. Fallon, J. Alghamdi, Y. Chiu, V. Sluming, T. Nurmikko, and A. Stancak, "Structural alterations in brainstem of fibromyalgia syndrome patients correlate with sensitivity to mechanical pressure," NeuroImage: Clinical, vol. 3, pp. 163-170, 2013.

[56] M. Ceko, M. C. Bushnell, M.-A. Fitzcharles, and P. Schweinhardt, "Fibromyalgia interacts with age to change the brain," NeuroImage: Clinical, vol. 3, pp. 249-260, 2013.

[57] C. Diaz-Piedra, M. A. Guzman, G. Buela-Casal, and A. Catena, "The impact of fibromyalgia symptoms on brain morphometry," Brain Imaging and Behavior, pp. 1-14, 2015.

[58] C. Destrieux, B. Fischl, A. Dale, and E. Halgren, "Automatic parcellation of human cortical gyri and sulci using standard anatomical nomenclature," NeuroImage, vol. 53, no. 1, pp. 1-15, 2010.

[59] B. A. Vogt, "Pain and emotion interactions in subregions of the cingulate gyrus," Nature Reviews Neuroscience, vol. 6, no. 7, pp. 533-544, 2005.

[60] B. A. Vogt, R. W. Sikes, and L. J. Vogt, "Anterior cingulate cortex and the medial pain system," in Neurobiology of Cingulate Cortex and Limbic Thalamus, pp. 313-344, Springer, 1993.

[61] A. K. P. Jones, B. Kulkarni, and S. W. G. Derbyshire, "Pain mechanisms and their disorders: imaging in clinical neuroscience," British Medical Bulletin, vol. 65, no. 1, pp. 83-93, 2003.

[62] V. Mylius, J. Reis, M. Kunz et al., "Modulation of electrically induced pain by paired pulse transcranial magnetic stimulation of the medial frontal cortex," Clinical Neurophysiology, vol. 117, no. 8, pp. 1814-1820, 2006.

[63] W.-K. Yoo, Y.-H. Kim, W.-S. Doh et al., "Dissociable modulating effect of repetitive transcranial magnetic stimulation on sensory and pain perception," NeuroReport, vol. 17, no. 2, pp. 141-144, 2006. 
[64] Y. Tamura, S. Okabe, T. Ohnishi et al., "Effects of 1-Hz repetitive transcranial magnetic stimulation on acute pain induced by capsaicin," Pain, vol. 107, no. 1-2, pp. 107-115, 2004.

[65] M. M. Heinricher, I. Tavares, J. L. Leith, and B. M. Lumb, "Descending control of nociception: specificity, recruitment and plasticity," Brain Research Reviews, vol. 60, no. 1, pp. 214225, 2009.

[66] I. Tracey and P. W. Mantyh, "The cerebral signature for pain perception and its modulation," Neuron, vol. 55, no. 3, pp. 377391, 2007.

[67] A. M. Abeles, M. H. Pillinger, B. M. Solitar, and M. Abeles, "Narrative review: the pathophysiology of fibromyalgia," Annals of Internal Medicine, vol. 146, no. 10, pp. 726-734, 2007.

[68] T. Schmidt-Wilcke and D. J. Clauw, "Pharmacotherapy in fibromyalgia (FM)-implications for the underlying pathophysiology," Pharmacology \& Therapeutics, vol. 127, no. 3, pp. 283-294, 2010.

[69] L. Gormsen, R. Rosenberg, F. W. Bach, and T. S. Jensen, "Depression, anxiety, health-related quality of life and pain in patients with chronic fibromyalgia and neuropathic pain," European Journal of Pain, vol. 14, no. 2, pp. 127.e1-127.e8, 2010.

[70] J. M. Glass, "Fibromyalgia and cognition," The Journal of Clinical Psychiatry, vol. 69, no. 2, pp. 20-24, 2008.

[71] R. R. Edwards, C. O. Bingham III, J. Bathon, and J. A. Haythornthwaite, "Catastrophizing and pain in arthritis, fibromyalgia, and other rheumatic diseases," Arthritis Care \& Research, vol. 55, no. 2, pp. 325-332, 2006.

[72] R. H. Gracely, M. E. Geisser, T. Giesecke et al., "Pain catastrophizing and neural responses to pain among persons with fibromyalgia," Brain, vol. 127, no. 4, pp. 835-843, 2004.

[73] A. Ploghaus, I. Tracey, J. S. Gati et al., "Dissociating pain from its anticipation in the human brain," Science, vol. 284, no. 5422, pp. 1979-1981, 1999.

[74] T. V. Sewards and M. A. Sewards, "Fear and power-dominance drive motivation: neural representations and pathways mediating sensory and mnemonic inputs, and outputs to premotor structures," Neuroscience \& Biobehavioral Reviews, vol. 26, no. 5, pp. 553-579, 2002.

[75] R. Luerding, T. Weigand, U. Bogdahn, and T. Schmidt-Wilcke, "Working memory performance is correlated with local brain morphology in the medial frontal and anterior cingulate cortex in fibromyalgia patients: structural correlates of pain-cognition interaction," Brain, vol. 131, no. 12, pp. 3222-3231, 2008.

[76] D. M. Amodio and C. D. Frith, "Meeting of minds: the medial frontal cortex and social cognition," Nature Reviews Neuroscience, vol. 7, no. 4, pp. 268-277, 2006.

[77] F. Å. Nielsen, D. Balslev, and L. K. Hansen, "Mining the posterior cingulate: segregation between memory and pain components," NeuroImage, vol. 27, no. 3, pp. 520-532, 2005.

[78] B. A. Vogt, G. R. Berger, and S. W. G. Derbyshire, "Structural and functional dichotomy of human midcingulate cortex," European Journal of Neuroscience, vol. 18, no. 11, pp. 3134-3144, 2003.

[79] A. Etkin, T. Egner, and R. Kalisch, "Emotional processing in anterior cingulate and medial prefrontal cortex," Trends in Cognitive Sciences, vol. 15, no. 2, pp. 85-93, 2011.

[80] B. A. Vogt, "Submodalities of emotion in the context of cingulate subregions," Cortex, vol. 59, pp. 197-202, 2014.

[81] B. Vogt, Cingulate Neurobiology and Disease, Oxford University Press, 2009.
[82] S. S. Can, A. Gencay-Can, and Z. Gunendi, "Validity and reliability of the clock drawing test as a screening tool for cognitive impairment in patients with fibromyalgia," Comprehensive Psychiatry, vol. 53, no. 1, pp. 81-86, 2012.

[83] R. Cánovas, I. León, M. D. Roldán, R. Astur, and J. M. Cimadevilla, "Virtual reality tasks disclose spatial memory alterations in fibromyalgia," Rheumatology, vol. 48, no. 10, pp. 1273-1278, 2009.

[84] S.-H. Kim, S.-H. Kim, S.-K. Kim, E. J. Nam, S. W. Han, and S. J. Lee, "Spatial versus verbal memory impairments in patients with fibromyalgia," Rheumatology International, vol. 32, no. 5, pp. 1135-1142, 2012.

[85] D. A. Gusnard, E. Akbudak, G. L. Shulman, and M. E. Raichle, "Medial prefrontal cortex and self-referential mental activity: relation to a default mode of brain function," Proceedings of the National Academy of Sciences of the United States of America, vol. 98, no. 7, pp. 4259-4264, 2001.

[86] P. Fransson and G. Marrelec, "The precuneus/posterior cingulate cortex plays a pivotal role in the default mode network: evidence from a partial correlation network analysis," NeuroImage, vol. 42, no. 3, pp. 1178-1184, 2008.

[87] S. Minoshima, B. Giordani, S. Berent, K. A. Frey, N. L. Foster, and D. E. Kuhl, "Metabolic reduction in the posterior cingulate cortex in very early Alzheimer's disease," Annals of Neurology, vol. 42, no. 1, pp. 85-94, 1997.

[88] R. Leech and D. J. Sharp, "The role of the posterior cingulate cortex in cognition and disease," Brain, vol. 137, no. 1, pp. 12-32, 2014.

[89] J. S. Damoiseaux, C. F. Beckmann, E. J. S. Arigita et al., "Reduced resting-state brain activity in the 'default network' in normal aging," Cerebral Cortex, vol. 18, no. 8, pp. 1856-1864, 2008.

[90] B. A. Vogt, L. Vogt, and S. Laureys, "Cytology and functionally correlated circuits of human posterior cingulate areas," $\mathrm{Neu}$ rolmage, vol. 29, no. 2, pp. 452-466, 2006.

[91] D. S. Margulies, J. L. Vincent, C. Kelly et al., "Precuneus shares intrinsic functional architecture in humans and monkeys," Proceedings of the National Academy of Sciences of the United States of America, vol. 106, no. 47, pp. 20069-20074, 2009.

[92] V. Napadow, L. LaCount, K. Park, S. As-Sanie, D. J. Clauw, and R. E. Harris, "Intrinsic brain connectivity in fibromyalgia is associated with chronic pain intensity," Arthritis \& Rheumatism, vol. 62, no. 8, pp. 2545-2555, 2010.

[93] M. N. Baliki, P. Y. Geha, A. V. Apkarian, and D. R. Chialvo, "Beyond feeling: chronic pain hurts the brain, disrupting the default-mode network dynamics," Journal of Neuroscience, vol. 28, no. 6, pp. 1398-1403, 2008.

[94] A. Kucyi, T. V. Salomons, and K. D. Davis, "Mind wandering away from pain dynamically engages antinociceptive and default mode brain networks," Proceedings of the National Academy of Sciences of the United States of America, vol. 110, no. 46, pp. 18692-18697, 2013.

[95] M.-H. Chang, J.-W. Hsu, K.-L. Huang et al., "Bidirectional association between depression and fibromyalgia syndrome: a nationwide longitudinal study," The Journal of Pain, vol. 16, no. 9, pp. 895-902, 2015.

[96] P. C. M. P. Koolschijn, N. E. M. van Haren, G. J. L. M. LensveltMulders, H. E. Hulshoff Pol, and R. S. Kahn, "Brain volume abnormalities in major depressive disorder: a meta-analysis of magnetic resonance imaging studies," Human Brain Mapping, vol. 30, no. 11, pp. 3719-3735, 2009.

[97] E. Bora, A. Fornito, C. Pantelis, and M. Yücel, "Gray matter abnormalities in Major Depressive Disorder: a meta-analysis of 
voxel based morphometry studies," Journal of Affective Disorders, vol. 138, no. 1-2, pp. 9-18, 2012.

[98] R. F. Smallwood, A. R. Laird, A. E. Ramage et al., "Structural brain anomalies and chronic pain: a quantitative meta-analysis of gray matter volume," The Journal of Pain, vol. 14, no. 7, pp. 663-675, 2013.

[99] A. May, "Structural brain imaging: a window into chronic pain," Neuroscientist, vol. 17, no. 2, pp. 209-220, 2011. 


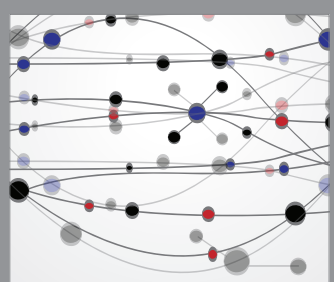

The Scientific World Journal
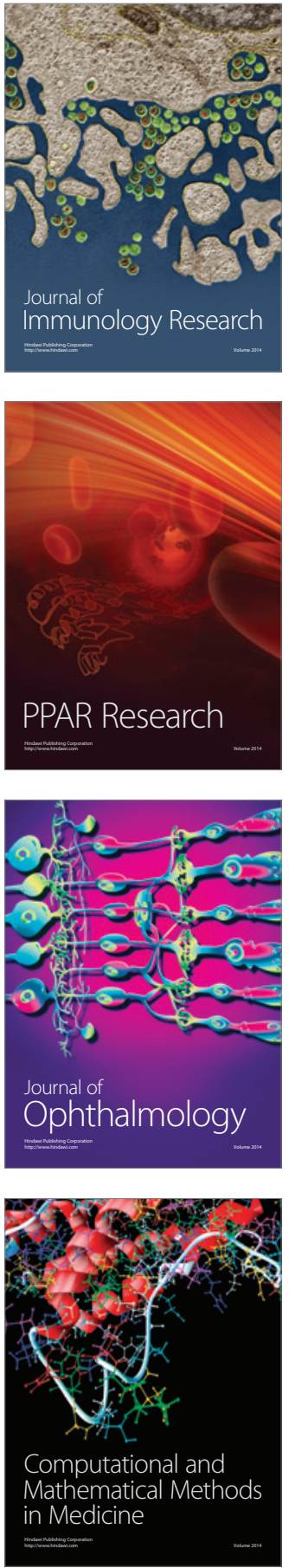

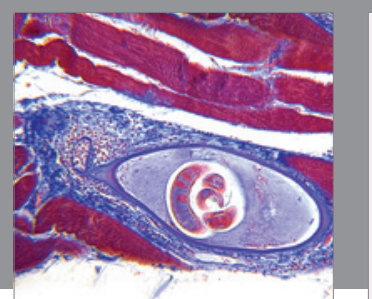

Gastroenterology Research and Practice

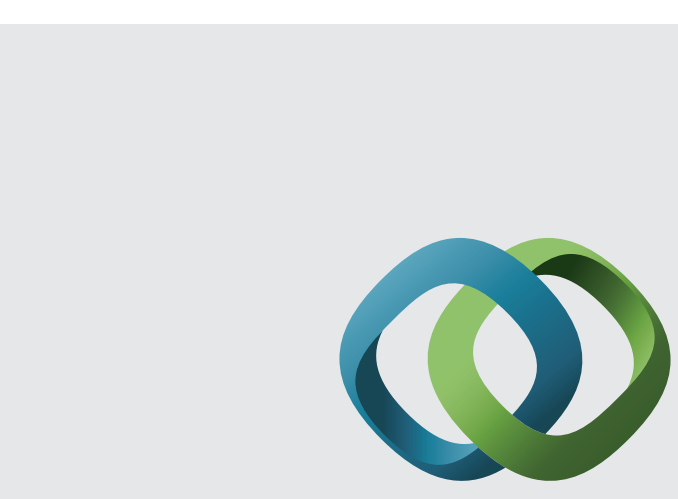

\section{Hindawi}

Submit your manuscripts at

http://www.hindawi.com
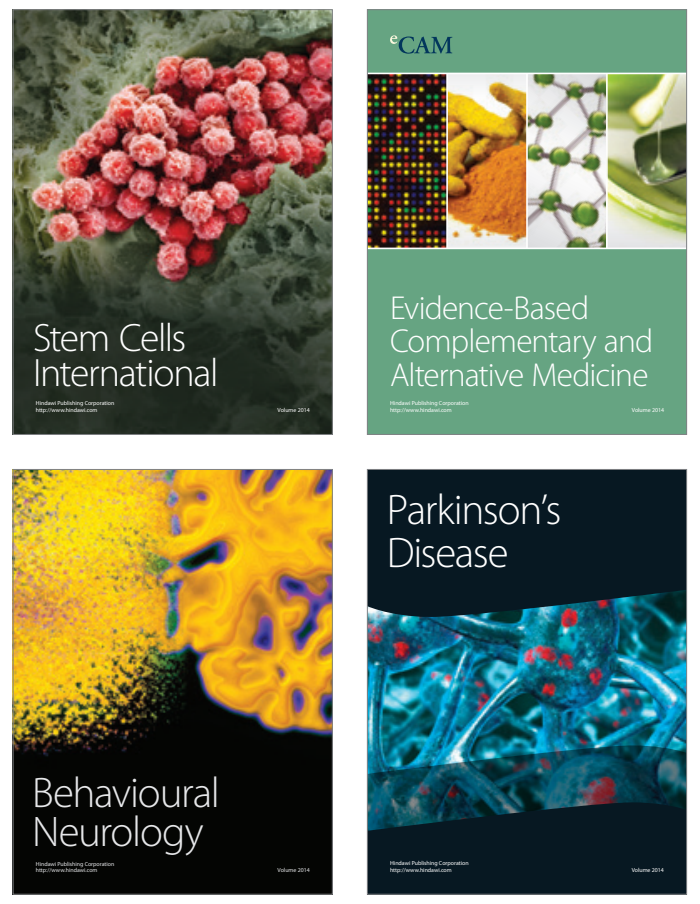
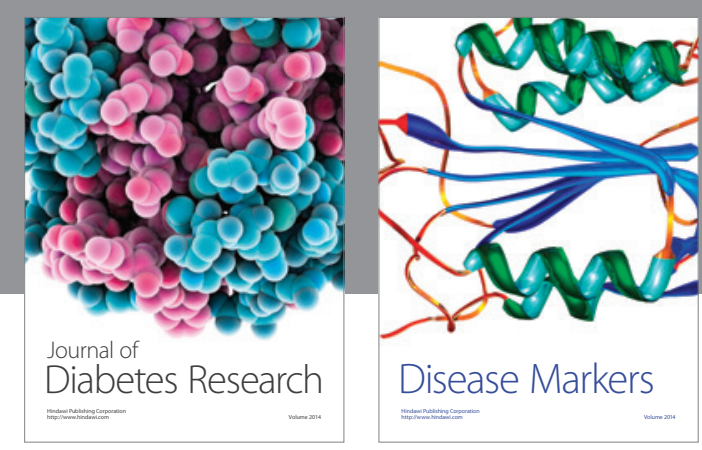

Disease Markers
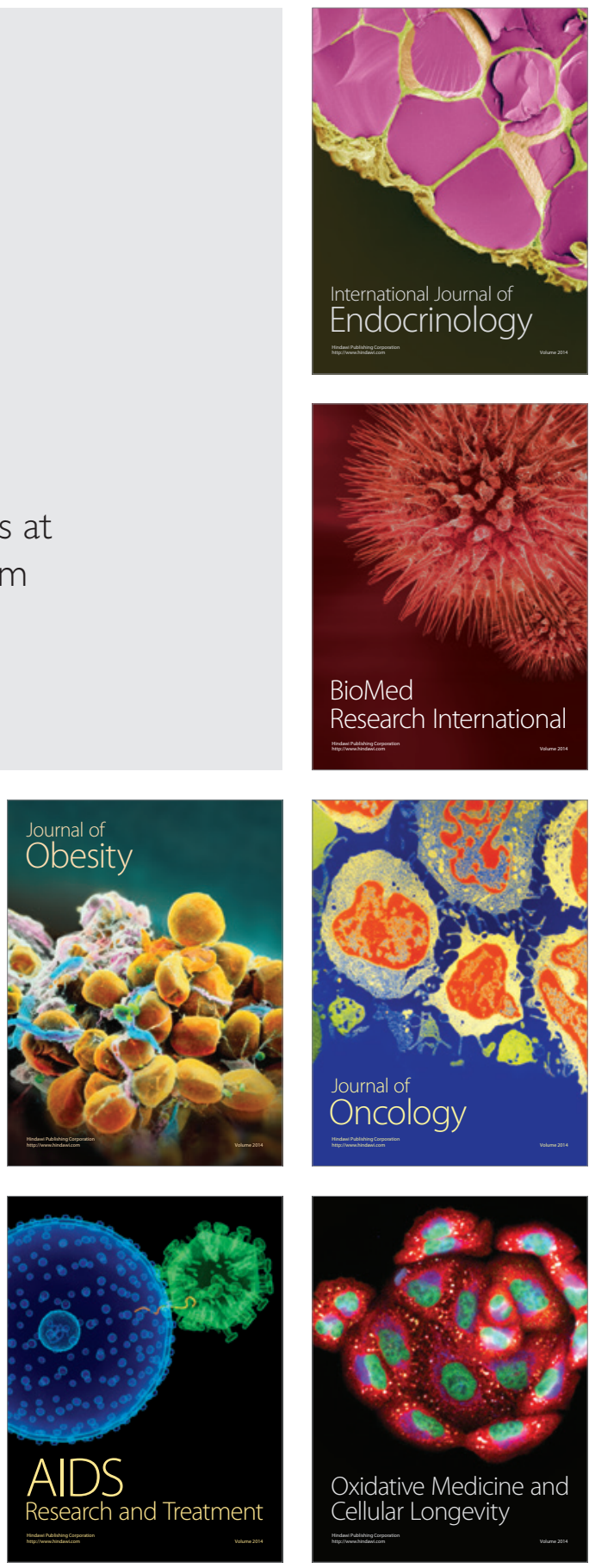